\title{
On an Analytical, Spatially-Varying, Point-Spread-Function
}

\author{
Lucien Roquette ${ }^{\dagger, *}$ \\ Matthieu Simeoni*, $†$ \\ Paul Hurley* \\ Adrien Besson ${ }^{\dagger}$ \\ lucien.roquette@epfl.ch \\ meo@zurich.ibm.com \\ pah@zurich.ibm.com \\ adrien.besson@epfl.ch \\ *IBM Zurich Research Laboratory, Rüschlikon; †École Polytechnique Fédérale de Lausanne (EPFL), Lausanne.
}

\begin{abstract}
The point spread function (PSF), namely the response of an ultrasound system to a point source, is a powerful measure of the quality of an imaging system. The lack of an analytical formulation inhibits many applications ranging from apodization optimization, array-design, and deconvolution algorithms. We propose to fill this gap through a general PSF derivation that is flexible with respect to the type of transmission (synthetic aperture, plane-wave, diverging-wave etc.), while faithfully capturing the spatially-variant blurring of the Tissue Reflectivity Function as caused by Delay-And-Sum reconstruction. We validate the derived PSF against simulation using Field II, and show that accounting for PSF spatial-variability in sparsebased deconvolution improves reconstruction.

Index Terms-Point-Spread-Function, deconvolution, image enhancement, ultrasonic image simulation, apodization design.
\end{abstract}

\section{BACKGROUND AND MOTIVATION}

In ultrasound (US) imaging, the finite bandwidth and aperture of transducer elements limits image resolution. This limitation on the resolving capability of the tissue reflectivity function (TRF) can be modelled explicitly by re-casting the radio-frequency (RF) image as the results of an operator between the point-spread function (PSF) and the TRF. In this sense, the PSF, defined as the response of the imaging method in presence of a single scatterer, contains the blurring due to the instrument, and is a powerful tool in assessing the equipment performance in terms of imaging quality.

Deconvolution methods use RF images to retrieve the TRF by accounting for the effect of PSF and prior knowledge of the image type. Many authors thus discuss deconvolution in conjunction with PSF assessment [1]-[3]. Two approaches to account for PSF blurring can be distinguished: deterministic [1], [2], [4] and blind deconvolution [3], [5].

Most deconvolution methods, blind or not, assume a spatially-invariant PSF model, primarily for computational purposes. In the blind-deconvolution context, [3], [5] argue that tissue-dependent attenuation and dispersive effects require the PSF to be estimated during the TRF computation. To avoid too complex an optimization, the PSF is usually assumed spatially-invariant across the imaging domain. In non-blind deconvolution [4], [6], a deterministic model of the PSF is obtained by means of simulation, such as Field II [7] or numerical approximation [1]. Again, the convenience of a spatiallyinvariant PSF avoids time-consuming repeated simulation.
In both cases, a spatial invariance assumption on the PSF hence leads to important computational gain, since the operator linking the TRF and RF image becomes a convolution, which can efficiently be implemented in the Fourier domain. This assumption can however have profound consequences on the quality of the recovered TRF images: in practice, the PSF can indeed vary quite dramatically across the imaging domain, leading to non-uniform recovery performances.

A few attempts have been made to account for spatiallyvarying PSFs [3]. However, they usually come at the cost of some simplifying assumptions. For example, and as mentioned in the introduction of [3], sub-segmenting the image leads to non-trivial "stitching" problems. In general, spatial-invariance necessitates deconvolution applications to small imaging domains, where the true PSF is not so badly approximated by the invariant approximation.

In this paper we obtain a PSF formulation based on a datamodel that relies on the Born approximation of the LippmannSchwinger equation (i.e., ignoring multiple scattering), propagation in an homogeneous medium, and point approximation of a transducer element. The derived formula results from the fundamental definition of a PSF, namely the resulting image when the medium is solely composed of a single scatterer. This PSF formula is analytical, and does not rely on numerical approximation of the wave-equation nor the well-known Field II simulator. We propose an application for deterministic spatially varying deconvolution. While ignoring the tissuedependant effect, we show that most of the spatial variability can be captured by considering the array layout and the waves natural propagation geometry. Furthermore, our analytical PSF could be a very convenient tool for assessing the expected resolution/contrast due to the array aperture, the number of elements, transducer bandwidth, the chosen excitation strategy (e.g. plane wave, diverging wave), or even the apodization method.

Section II presents an overview of the data-model, which was closely inspired by [8]. From a time-signals model and a natural definition of the PSF for ultrasound imaging, we derive an analytical formula encompassing any imaging mode. Section IV demonstrates first how close the analytical formula is from Field II simulation for the case of diverging waves. We then use a spatially-invariant sparse-promoting deconvolution of a point-based phantom image, obtained via our PSF, show- 
ing its poor reconstruction capability across the entire imaging plane.

\section{DATA-MODEL AND PSF FORMULA}

The raw-data received at the $n$th transducer is linked to the TRF, $\chi \in L^{2}(\Omega)$ with the pulse-echo impulse response model [7], which is a rather generic model. The notation used is introduced in Figure 1. The round-trip time-of-flight $\tau\left(\vec{r}, \vec{p}_{i}, \vec{p}_{j}\right)$ from the transducer $\vec{p}_{i}(i=1, \ldots, M)$ to the medium point $\vec{r}$ and back to $\vec{p}_{j}$ is decomposed into two contributions: $\tau\left(\vec{r}, \vec{p}_{i}, \vec{p}_{j}\right)=t_{T x}\left(\vec{r}, \vec{p}_{i}\right)+\left|\vec{r}-\vec{p}_{j}\right| / c$, where $c$ is the speed of sound in the medium. Without loss of generality, we restrict our attention to the transmission mode where the round-trip time-of-flight only depends on a single transducer element in the array. For instance, the following techniques satisfy such a restriction:

- Plane wave (wavefront parallel to the array), $\tau\left(\vec{r}, \vec{p}_{i}\right)=$ $\left\langle\vec{e}_{z}, \vec{r}\right\rangle / c+\left|\vec{r}-\vec{p}_{i}\right| / c$

- Diverging wave, $\tau\left(\vec{r}, \vec{p}_{i}\right)=\left|\vec{r}-\vec{r}_{n}\right| / c+\left|\vec{r}-\vec{p}_{i}\right| / c$, where $\vec{r}_{n}$ is a virtual source point;

- Synthetic Focusing Aperture Technique (SAFT), $\tau\left(\vec{r}, \vec{p}_{i}\right)=2\left|\vec{r}-\vec{p}_{i}\right| / c$.

The pulse function $p(t)=e(t) \circledast h_{e l}(t) \circledast h_{e l}(t)^{1}$ results from the transducer electro-mechanical impulse response, $h_{e l}(t)$, at emission and recepetion (assumed to be the same) and the electrical excitation imposed. The time signal recorded at sensor $\vec{p}_{i}(i=1, \ldots, M)$ can then be modelled as

$$
R_{i}(t)=\int_{\Omega} b\left(\vec{r}, \vec{p}_{i}\right) \frac{p\left\{t-\tau\left(\vec{r}, \vec{p}_{i}\right)\right\}}{\left|\vec{r}-\vec{p}_{i}\right|} \chi(\vec{r}) d \vec{r}, \quad t \in[0, T],
$$

where $T$ is the final acquisition time and $b\left(\vec{r}, \vec{p}_{i}\right)$ is the spatial sensitivity of the transducer element. This data model is simpler than the one implemented in Field II [7]. In particular, it neglects the array pattern, and the element directivity is simplified using a point approximation of the transducer through the well-acknowledged formula [9]. For the sake of simplicity, in the following derivation the spatial sensitivity is assumed to be equal to one.

The RF image is obtained by applying the Delay-and-Sum (DAS) reconstruction method to the time-signal $R_{i}(t)$, and will denote the estimated TRF $\hat{\chi}^{\mathrm{DAS}}(\vec{r})$. Fundamentally, we will define the PSF as the bivariate kernel satisfying

$$
\hat{\chi}^{\mathrm{DAS}}(\vec{r})=\int_{\Omega} \operatorname{PSF}(\vec{r}, \vec{s}) \chi(\vec{s}) d \vec{s},
$$

where $\chi(\vec{s})$ is the true TRF. If the medium is composed of a single scatterer located at $\vec{r}_{0} \in \Omega$, i.e., $\chi(\vec{r})=\delta\left(\vec{r}-\vec{r}_{0}\right)$ then the RF image corresponds to the PSF: $\hat{\chi}^{\mathrm{DAS}}(\vec{r})=\operatorname{PSF}\left(\vec{r}, \vec{r}_{0}\right)$. The PSF operator introduced in (2) may model both the spatially variant and invariant cases. In the latter, the PSF operator has the property $\operatorname{PSF}(\vec{r}, \vec{s})=\operatorname{PSF}(\vec{r}-\vec{a}, \vec{s}-\vec{a})$. In particular, this shift-invariance property permits us to recast (2) as the convolution $\hat{\chi}^{\mathrm{DAS}}(\vec{r})=\operatorname{PSF}(\cdot, 0) \circledast_{2 D} \chi(\cdot)$.

$$
{ }^{1} f \circledast g(t):=\int_{\mathbb{R}} f(t-\tau) g(\tau) d \tau
$$

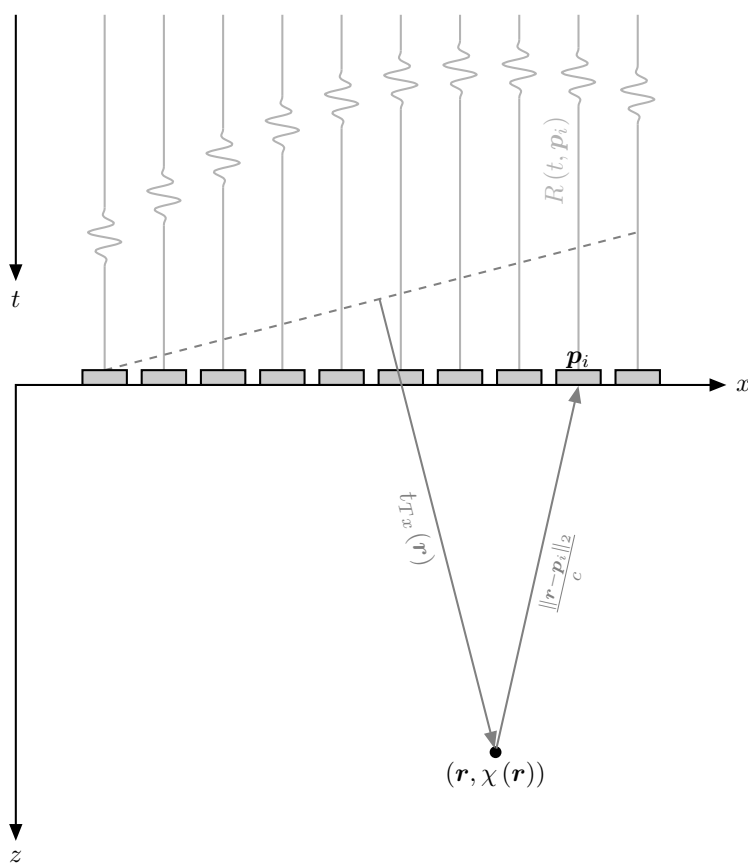

Fig. 1: Schematic setting for ultrasound imaging.

The DAS estimate at a point $\vec{r} \in \Omega$ corresponds to evaluating the time signal at an appropriate round-trip timeof-flight:

$$
\hat{\chi}^{\mathrm{DAS}}(\vec{r})=\sum_{i=1}^{M} w_{i} R_{i}\left\{\tau\left(\vec{r}, \vec{p}_{i}\right)\right\}
$$

where the weights $w_{i}(i=1, \ldots, M)$ account for any potential apodization strategy on receive. As previously mentioned, the PSF formula can be obtained by injecting the signal obtained from a single-scatterer medium,

$$
R_{i}(t)=p\left\{t-\tau\left(\vec{r}_{0}, \vec{p}_{i}\right)\right\} /\left|\vec{r}_{0}-\vec{p}_{i}\right|, \quad t \in[0, T],
$$

into the DAS estimate formula given by (3), from which the expression of the PSF simply becomes:

$$
\operatorname{PSF}\left(\vec{r}, \vec{r}_{0}\right)=\sum_{i=1}^{M} w_{i} p\left\{\tau\left(\vec{r}, \vec{p}_{i}\right)-\tau\left(\vec{r}_{0}, \vec{p}_{i}\right)\right\} /\left|\vec{r}_{0}-\vec{p}_{i}\right| .
$$

It can be seen that this formulation satisfies the definition (2) by plugging in the RF image definition (3) into the data-model (1).

Furthermore, note that the PSF is functionally defined over $\Omega$, and may hence be evaluated on any grid, which turns out to be extremely useful for performing a spatially-variant deconvolution as depicted in Section IV-B.

\section{Formulation OF THE DECONVOLUTION PROBLEM}

The proposed PSF formulation is compatible with any stateof-the-art deconvolution method. For this paper we focus on an example sparse-based method similar to [6], [8]. The aim is to confirm the intuition that using a more accurate characterization of the PSF, even with the above-mentioned simplistic 
model, leads to a better reconstruction than a spatially invariant PSF.Consider an RF image $\hat{\chi}^{D A S} \in \mathbb{R}^{N_{x} N_{y}}$ defined over a spatial grid of $\Omega$ :

$$
\Omega_{\text {Grid }}=\left\{\left(x_{i}, y_{k}\right) \in \Omega: i=1, \ldots, N_{x} \quad k=1, \ldots, N_{y}\right\} .
$$

This image is linked to the TRF evaluated on $\Omega_{\text {Grid }}$ by

$$
\hat{\chi}^{D A S}=H \chi+\epsilon,
$$

where $H: \mathbb{R}^{N_{x} N_{y}} \rightarrow \mathbb{R}^{N_{x} N_{y}}$ is the forward-operator linking the actual RF image to the TRF, and $\epsilon$ is the noise induced by model inaccuracy due to its underlying simplifying assumptions and the grid discretization. The TRF image is obtained by solving the optimization problem:

$$
\min _{\chi \in \mathbb{R}^{N_{x} N_{y}}}\|\chi\|_{l_{1}} \quad \text { s-t. } \quad\left\|\hat{\chi}^{D A S}-H \chi\right\|_{l_{2}} \leq \lambda,
$$

where $\lambda>0$ trades-off the data-fidelity criterion against the sparsity promoting one. The optimization problem is solved using the ADMM algorithm [10], and the results are presented in Section IV-B.

\section{RESUlts}

For illustration, we focused on a point-like phantom similar to the one used during IEEE IUS 2016 for the PICMUS challenge [11]. The medium is considered to be insonified by a diverging wave generated by a phased-array probe of 64 elements. Each transducer has a central frequency $f_{0}=4 \mathrm{Mhz}$, a bandwidth of 0.4 and a sampling frequency of $f_{s}=10 \mathrm{Mhz}$. The array pitch is $d=\lambda_{0} / 2$ and the electro-mechanical impulse response model is the Gaussian $h_{e l}(t)=e^{-\alpha t^{2}} \cos \left(2 \pi t f_{0}\right)$. The RF image computed from Field II simulations, generated in the described experimental settings, is obtained by applying DAS (3) with a spline interpolation between the discrete time-samples.

\section{A. Validation of the PSF formula against Field II simulation}

In Figure 2, the DAS image obtained from Field II data (Figure 2b) is compared to the one computed with the PSF formula (Figure 2c). This latter consists of applying (2) to $\chi(\vec{r})=\sum_{q=1}^{Q} \delta\left(\vec{r}-\vec{r}_{q}\right)$, where $\vec{r}_{q}$ corresponds to the location of the point scatterers defining the phantom in Figure $2 \mathrm{a}$.

Comparing Figure $2 \mathrm{~b}$ and $2 \mathrm{c}$ shows how similar the image obtained by the analytical formula is to the Field II-based DAS one. In particular, we observe that the spatial-variability of the PSF over the imaging plane is well accounted for. Due to the diverging wave, the PSF rotates over itself when one moves from the center $\left(x_{0}=0\right)$ to the right or the left along the lateral dimension. Along the depth direction the PSF tends to shrink over itself, implying a lost of lateral resolution when considering points deeper in the tissue.

In addition to the illustrated diverging wave case, the spatialvariability of the PSF arises in many others imaging modes whenever the domain $\Omega$ is sufficiently large.

\section{B. Spatially-varying deconvolution}

Figures $2 \mathrm{~b}$ and $2 \mathrm{c}$ show the PSF spatial-variability across the imaging plane. The aim here is to show that accounting for it is of primary importance when it comes to deconvolving the RF image. For the sake of simplicity, we considered an RF image generated by our own PSF model following (5) with no noise. The forward operator $H: \mathbb{R}^{N_{x} N_{y}} \rightarrow \mathbb{R}^{N_{x} N_{y}}$ used to compute the RF image is defined through PSF formula (4) evaluated on a specified grid:

$$
H_{i j}=\operatorname{PSF}\left(\vec{r}_{i}, \vec{r}_{j}\right), \quad i, j=1, \ldots, N_{x} N_{y} .
$$

Figures $3 \mathrm{a}$ and $3 \mathrm{~b}$ show the results when applying a spatiallyinvariant deconvolution on the point-scatterer RF image obtained in similar conditions to those in Section IV-A. To avoid computation on too large a grid we restricted ourself to a $3 \mathrm{~cm}$ depth image instead of the $5 \mathrm{~cm}$ in Section IV-A, leading to $N_{x}=N_{y}=100$. We note that in Figures $3 \mathrm{a}$ and $3 \mathrm{~b}$ that the scatterer near $\vec{r}_{c}$ (where the PSF is computed) is always accurately recovered independently of the model fidelity parameter $\lambda$. Figure $3 \mathrm{a}$ illustrates that choosing a high-model fidelity, while the model does not account the spatial variability, leads to a poor deconvolution of the TRF. In contrast, Figure $3 \mathrm{~b}$ shows a large flexibility in the model specification implies a loss of information as the four scatterers the most distant from $\vec{r}_{c}$ disappear. This behaviour is expected since the spatially-invariant assumption becomes too crude further away from $\vec{r}_{c}$, and hence demonstrates the importance of accounting for it when it comes to deconvolution.

In Figure 3c, we apply the suggested spatially-varying deconvolution using the forward operator computed according to (6). We observe that the TRF is perfectly recovered uniformly over the plane. This is not surprising since we are using the model (6) to convolve the TRF and obtained the RF image. Note that the results in Figure $3 \mathrm{c}$ are identical for a wide range of $\lambda$, i.e., the deconvolution is less sensitive to it than in the spatially-invariant case. This behavior is due to the adequacy between the PSF model and the RF image, and is a highly desired feature in practice so as to avoid the computationally expensive search for an appropriate $\lambda$.

\section{COnClusions}

We introduced a new formulation of the PSF as the kernel of the integral operator acting on the TRF in order to form the RF image (2). From this perspective, we derived an analytical formula for it that encompasses any emitting wave strategy, array geometry, transducer bandwidth, electrical excitation or apodization method. This explicit PSF was shown to very accurately characterize the one obtained by Field II simulation. Moreover, we demonstrated the application of the analytical PSF to spatially-varying deterministic deconvolution. In particular, we illustrated the lack of reconstruction capability when the model does not account for the spatial variation. In addition to the deconvolution context, we believe that an analytical understanding of the PSF could lead to many other applications ranging from array design to apodization optimization. 


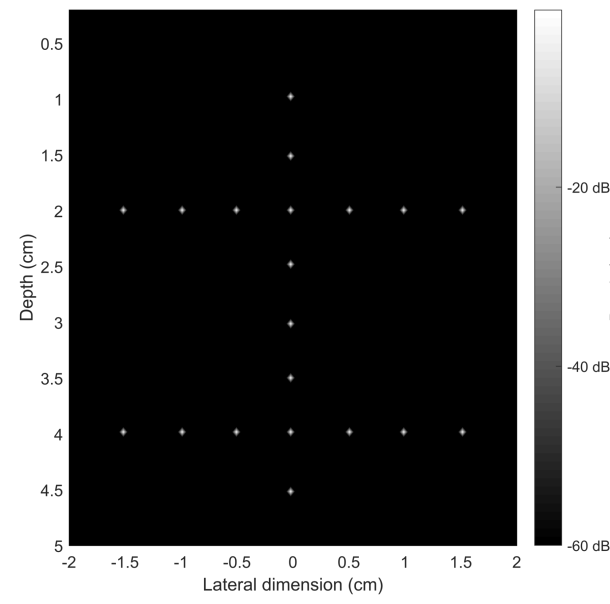

(a)

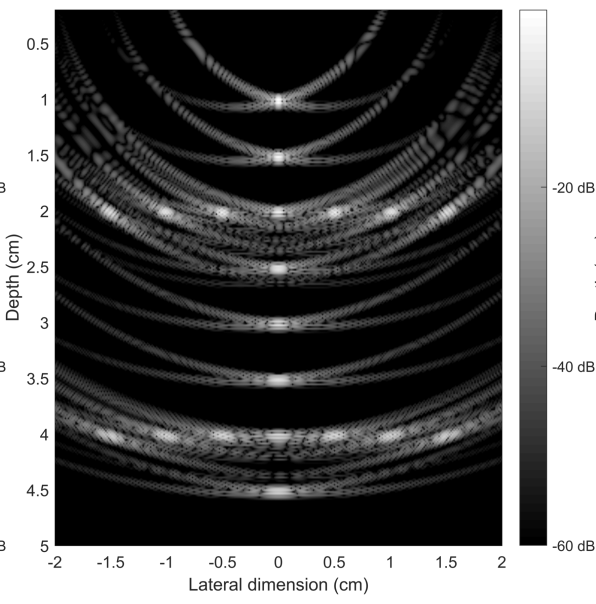

(b)

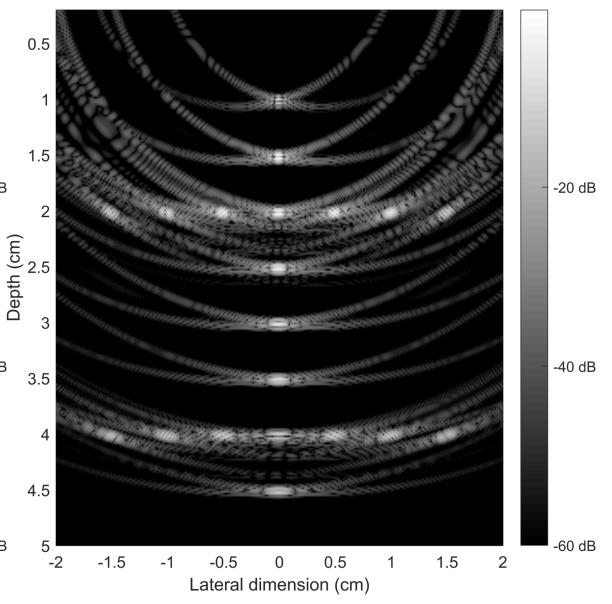

(c)

Fig. 2: (a)-(c): Comparison between Field II simulations of the PSF and the results obtained with the analytical formula. (a) Point-reflectors phantom used. (b) B-mode image of the point-reflectors obtained with DAS algorithm applied on element raw-data simulated with Field II; (c) B-mode image obtained with the PSF formula (4) summed over all the point reflectors in (a).

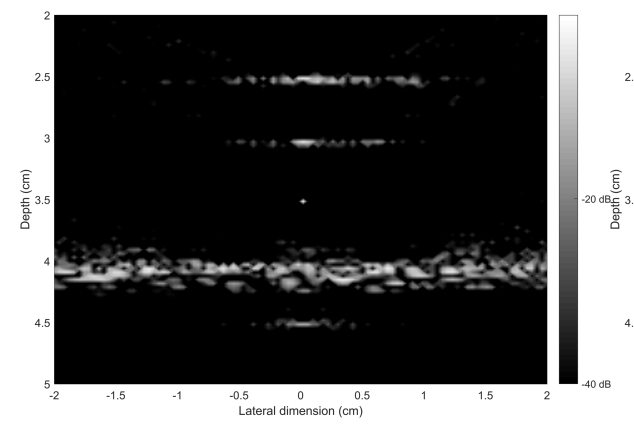

(a)

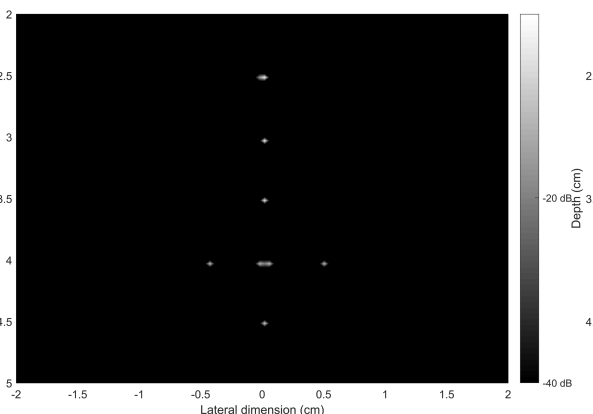

(b)

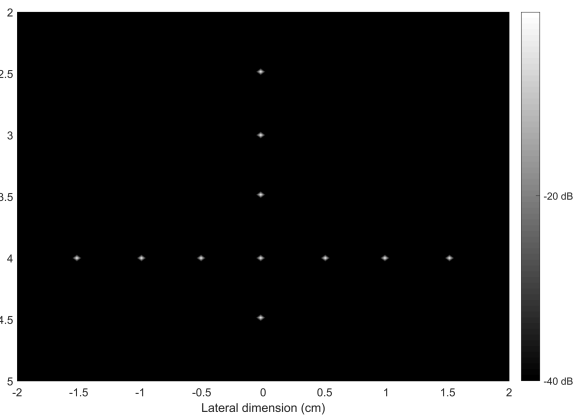

(c)

Fig. 3: The estimated TRF through deconvolution for different amount of sparsity promotion, or equivalently of model fidelity with a spatially invariant (a)-(b) and variant (c) versions of the PSF. The original RF image is given in Figure 2c but restricted to depth-region between 2 and $5 \mathrm{~cm}$. (a) Spatially-invariant: high model fidelity (small $\lambda$ ) (b) Spatially-invariant: low model fidelity (high $\lambda$ ) (c) Spatially-varying: high model fidelity.

\section{REFERENCES}

[1] R. Rangarajan, C. Krishnamurthy, and K. Balasubramaniam, "Ultrasonic Imaging using a Computed Point Spread Function," IEEE transactions on ultrasonics, ferroelectrics, and frequency control, vol. 55, no. 2, 2008.

[2] C. Dalitz, R. Pohle-Frohlich, and T. Michalk, "Point Spread Functions and Deconvolution of Ultrasonic Images," IEEE transactions on ultrasonics, ferroelectrics, and frequency control, vol. 62, no. 3, pp. 531-544, 2015.

[3] O. V. Michailovich, "Non-stationary Blind Deconvolution of Medical Ultrasound Scans," in SPIE Medical Imaging. International Society for Optics and Photonics, 2017, pp. $101391 \mathrm{C}-101391 \mathrm{C}$.

[4] H.-C. Shin, R. Prager, J. Ng, H. Gomersall, N. Kingsbury, G. Treece, and A. Gee, "Sensitivity to Point-Spread Function Parameters in Medical Ultrasound Image Deconvolution," Ultrasonics, vol. 49, no. 3, pp. 344 357, 2009.

[5] O. Michailovich and A. Tannenbaum, "Blind Deconvolution of Medical Ultrasound Images: A Parametric Inverse Filtering Approach," IEEE Transactions on Image Processing, vol. 16, no. 12, pp. 3005-3019, 2007.

[6] R. E. Carrillo, A. Besson, M. Zhang, D. Friboulet, Y. Wiaux, J.P. Thiran, and O. Bernard, "A Sparse Regularization Approach for
Ultrafast Ultrasound Imaging," in Ultrasonics Symposium (IUS), 2015 IEEE International, 2015, pp. 1-4.

[7] J. A. Jensen, "Simulation of Advanced Ultrasound Systems using Field II," in Biomedical Imaging: Nano to Macro, 2004. IEEE International Symposium on, 2004, pp. 636-639.

[8] G. David, J.-1. Robert, B. Zhang, and A. F. Laine, "Time Domain Compressive Beam Forming of Ultrasound Signals," The Journal of the Acoustical Society of America, vol. 137, no. 5, pp. 2773-2784, 2015.

[9] A. Selfridge, G. Kino, and B. Khuri-Yakub, "A Theory for the Radiation Pattern of a Narrow-Strip Acoustic Transducer," Applied Physics Letters, vol. 37, no. 1, pp. 35-36, 1980.

[10] S. Boyd, N. Parikh, E. Chu, B. Peleato, and J. Eckstein, "Distributed Optimization and Statistical Learning via the Alternating Direction Method of Multipliers," Found. Trends Mach. Learn., vol. 3, no. 1, pp. 1-122, Jan. 2011. [Online]. Available: http://dx.doi.org/10.1561/2200000016

[11] H. Liebgott, A. Rodriguez-Molares, F. Cervenansky, J. A. Jensen, and O. Bernard, "Plane-Wave Imaging Challenge in Medical Ultrasound," in Ultrasonics Symposium (IUS), 2016 IEEE International, 2016, pp. 1-4. 